\title{
Édouard Glissant, L'imaginaire des langues. Entretiens avec Lise Gauvin (1991-2009)
}

\section{Carminella Biondi}

\section{(2) OpenEdition}

\section{Journals}

\section{Edizione digitale}

URL: http://journals.openedition.org/studifrancesi/5790

DOI: 10.4000/studifrancesi.5790

ISSN: 2427-5856

\section{Editore}

Rosenberg \& Sellier

\section{Edizione cartacea}

Data di pubblicazione: 1 septembre 2011

Paginazione: 465

ISSN: 0039-2944

\section{Notizia bibliografica digitale}

Carminella Biondi, «Édouard Glissant, L'imaginaire des langues. Entretiens avec Lise Gauvin (1991-2009)», Studi Francesi [Online], 164 (LV | II) | 2011, online dal 30 novembre 2015, consultato il 08 janvier 2021. URL: http://journals.openedition.org/studifrancesi/5790; DOI: https://doi.org/10.4000/studifrancesi. 5790

Questo documento è stato generato automaticamente il 8 janvier 2021.

\section{(c)}

Studi Francesi è distribuita con Licenza Creative Commons Attribuzione - Non commerciale - Non opere derivate 4.0 Internazionale. 


\title{
Édouard Glissant, L'imaginaire des langues. Entretiens avec Lise Gauvin (1991-2009)
}

\author{
Carminella Biondi
}

\section{NOTIZIA}

ÉDOUARD GLISSANT, L'imaginaire des langues. Entretiens avec Lise Gauvin (1991-2009), Paris,

Gallimard, 2010, $121 \mathrm{pp}$.

1 In ambito letterario l'entretien è considerato un genere minore, anche se richiede, per assolvere bene il suo compito, notevole abilità da parte dell'intervistatore e capacità di sintesi non banalizzante da parte dell'autore. Ma quando l'intervistato è uno dei più grandi scrittori (appena scomparso) del nostro tempo e l'intervistatrice una scrittrice che è anche critico letterario, non si può più parlare di genere minore ma di un piccolo capolavoro nato dalla collaborazione, o meglio, per adottare il linguaggio glissantiano, dalla "relazione" di due intelligenze che riflettono insieme sui problemi del nostro tempo e sul ruolo della letteratura. Il volume raccoglie sei interviste che coprono un arco temporale di quindici anni, i più fecondi di Glissant sul piano della riflessione teorica, perché hanno visto la pubblicazione di una serie di saggi o di romanzi-saggio che hanno contribuito all'elaborazione e alla diffusione di una poetica e di un modello narrativo fra i più originali a cavallo fra il secondo e il terzo millennio, a cominciare da Poétique de la Relation (1990) per finire con Philosophie de la relation. Poésie en étendue (2009), passando per il romanzo Tout-monde (1993), traduzione sul piano narrativo di una poetica che prevede un'apertura sulla totalità-mondo e una provocatoria contaminazione dei generi letterari. Le sei interviste indicano bene, per un verso l'evoluzione o forse meglio l'approfondimento di alcune idee fondanti della poetica di Glissant, quali creolizzazione, identità-rizoma versus identità a radice unica, Relazione con i suoi correlati di opacità e di trasparenza, caos-mondo, Tutto-mondo, pensiero 
arcipelico o del tremore che rifiuta le compattezze sistemiche per mettersi in sintonia con un mondo in evoluzione, non più chiuso su alcune delle sue parti dominanti ma in cerca della sua fragile totalità, e infine il pensiero o meglio l'immaginario delle lingue che ha un ruolo fondante in questo processo di appercezione e di scrittura del mondo. $\grave{E}$ quest'ultimo il filo rosso che lega le diverse interviste, e non è un caso perché Lise Gauvin, come Glissant, ha dedicato una larga parte dei suoi saggi critici, e in ogni caso quelli che hanno suscitato più consensi e dibattiti, alla riflessione sul problema delle lingue e in particolare della lingua francese, a cominciare da Rabelais per finire alle più spinte sperimentazioni in area francofona: L'écrivain francophone à la croisée des langues (1997 e 2006), Langagement. L'écrivain et la langue au Québec (2000), La fabrique de la langue. De François Rabelais à Réjan Ducharme (2004). Lise Gauvin, soffermandosi sull'atteggiamento dello scrittore extra-esagonale di fronte alla lingua francese, è arrivata alla conclusione che la riflessione per lui imprescindibile sulla lingua da utilizzare lo porta a dotarsi di una "surconscience linguistique» che diventa uno strumento potente di creazione e di innovazione, innanzitutto ma non solo, linguistica. È una definizione che ha avuto fortuna, ma a noi interessa in questo contesto per dire che le interviste riunite nel volume sono il risultato felice di due interessi convergenti e di una lunga riflessione sia da parte dell'intervistato che dell'intervistatore. Precedute da un Avant-dire di Lise Gauvin dal titolo Édouard Glissant: une pensée archipélique, le interviste sono, in ordine cronologico: L'imaginaire des langues (1993), (pp.11-34); L'écrivain et le souffle du lieu (1995), (pp. 35-57); Faire le guet du monde (2000), (pp. 59-72); Repenser l'utopie (2005), (pp. 73-85); De la beauté comme connivence (2006), (pp. 87-102); Passages des langues et territoires du roman (2009), (pp. 103-117). Nella breve introduzione in cui, come viatico alla lettura delle interviste che seguono, abbozza una sintesi del pensiero di Glissant, Lise Gauvin, lei stessa scrittrice e molto sensibile dunque alla qualità del linguaggio, coglie perfettamente il tono, la ricchezza e il potere seduttivo della scrittura glissantiana: «il n'y a de pensée véritable, selon lui, que celle qui rejoint le poème, celui-ci étant "la seule dimension de vérité ou de permanence ou de deviance qui relie les présences du monde" [...]. C'est en poète que Glissant développe cette pensée archipélique qui est au cœur de ses essais [...]. C'est en poète qu'il s'adresse à Barack Obama [...]. C'est en poète encore qu'il s'attaque aux transparences d'un universel généralisant [...]. C'est en poète qu'il aborde le devenir du Tout-monde, qui ne serait pas lié à celui d'une langue unique [...] mais à la multiplicité des idiomes» (p. 8).

2 Non mi soffermerò sulle singole interviste, alcune delle quali sono conosciute anche in Italia perché pubblicate dalla rivista «Francofonia» (si tratta delle penultime due, uscite rispettivamente nel 2006 e 2007, nn. 50 e 52), ma l'ultima, ancora inedita prima di essere inserita nella raccolta, merita attenzione, innanzitutto perché è una delle ultime interviste dello scrittore, ma anche perché unisce alla riflessione sulle lingue, ed in particolare sulla loro evoluzione e sul pericolo di sopraffazione da parte di alcune lingue più forti, uno spunto di dibattito sul presente ed il futuro di quello che è stato il genere dominante del Novecento, vale a dire il romanzo. A proposito delle lingue, Glissant si dice fiducioso sul mantenimento della loro feconda pluralità: «les langues vont se diversifier, se multiplier et s'archipéliser, et je crois que les langues qui auront la chance de ne pas disparaitre quand cette situation va arriver, elles vont embellir» (p. 110). A proposito del romanzo, invece, che meriterebbe come afferma in apertura di risposta, non due o tre ma almeno cento pagine, è più possibilista sulla sua fine, almeno sulla fine del vecchio modello occidentale che si è concepito non tanto o non solo come una tecnica, ma come «la croyance qu'on peut dire l'histoire, le monde, qu'on est les 
seuls à pouvoir le dire parce qu'on est les seuls à le contrôler» (p. 115). Questo non significa che non possa essere sostituito da nuovi o da antichissimi modelli di scrittura e ancora una volta, in conclusione di intervista, Glissant ribadisce la sua fiducia nella forza, anche vitale, della poesia che potrebbe in futuro farsi carico di mantenere in vita la letteratura. Alla domanda su che cosa sostituirà in futuro il romanzo, Glissant risponde di non saperlo, ma a proposito della poesia afferma: «La poésie a toujours remplacé tout. La poésie a toujours été le nœud de la littérature. Parce que la poésie est le seul art littéraire qui dit sans dire tout en disant» (p. 116). 